\title{
ANÁLISE DA CONCENTRAÇÃO E DO PH DE DIFERENTES MARCAS COMERCIAIS DE HIPOCLORITO DE SÓDIO
}

\section{ANALYSIS OF CONCENTRATION AND PH OF VARIOUS TRADEMARKS OF SODIUM HYPOCHLORITE}

\author{
Izabel Coelho Gomes Camões* \\ Lílian Ferreira Freitas* \\ Cristina Nunes Santiago"* \\ Cynthia Cristina Gomes"*... \\ Fernanda Valle de Menezes
}

\section{RESUMO}

\begin{abstract}
Introdução: Esta pesquisa, realizada na Universidade Federal Fluminense, se propõe a avaliar possível variação na concentração e no pH de diferentes marcas comerciais de hipoclorito de sódio durante 4 semanas. Foram utilizadas quatro marcas comerciais: Soda Clorada lodontosul 5\%, Soda Clorada Asfer 2,5\%, Água Sanitária Max Globo 2,0\% a 2,5\% e Solução manipulada de Hipoclorito de Sódio 5,25\% (Crystal Pharm). Método: Para a avaliação tanto do $\mathrm{pH}$ quanto da concentração do hipoclorito de sódio para cada uma das marcas comerciais diferentes, cada solução foi medida durante 4 semanas. As soluções eram avaliadas quanto ao $\mathrm{pH}$ a cada semana e a concentração a cada 2 semanas. Resultados: Os resultados mostraram, em relação ao pH, que as soluções avaliadas se mantiveram constantes durante a pesquisa. Em relação à concentração, somente a água sanitária Max Globo se manteve fiel, de acordo com a concentração estabelecida pelo fabricante.

DESCRITORES: Hipoclorito de Sódio • Concentração de íons de hidrogênio
\end{abstract}

\section{A B S TRACT}

Introduction: This study aims to assess possible changes in concentration and $\mathrm{pH}$ of different brands of sodium hypochlorite for 4 weeks. We used four brand names: Soda Chlorinated lodontosul 5\%, Soda Chlorinated Asfer 2,5\%, Sodium Hypochlorite Max Globo 2,0\% a 2,5\% and manipulated in solution Sodium Hypochlorite 5,25\% (Crystal Pharm). Method: For the evaluation of both the $\mathrm{pH}$ and the concentration of sodium hypochlorite for each of different commercial brands, each solution was measured for 4 weeks. The solutions were evaluated every week for $\mathrm{pH}$ and for concentration every 2 weeks. Results: The results showed in relation to $\mathrm{pH}$ that the evaluated solutions remained constant during the search. Regarding the merger, only Bleach Max Globe remained faithful, according to the concentration stated by the manufacturer. DESCRIPTORS: Sodium hypochlorite • Hydrogen-ion concentration

\footnotetext{
* Doutora em Odontologia - UFRJ. Prof. da Disciplina de Endodontia do Departamento de Odontoclínica da Universidade Federal Fluminense (UFF). Niterói, Rio de Janeiro, Brasil. e-mail: icamões@netbotanic.com.br

** Doutora em Endodontia UERJ. Prof. da Disciplina de Endodontia do Departamento de Odontoclínica da Universidade Federal Fluminense(UFF). Niterói, Rio de Janeiro, Brasil. e-mail: lilianffreitas@uol.com.br

*** Mestre em Odontologia Social (UFF). Prof. da Disciplina de Endodontia do Departamento de Odontoclínica da Universidade Federal Fluminense(UFF). Niterói,Rio de Janeiro, Brasil. e-mail: cris.nsantiago@yahoo.com.br

**** Doutora em Endodontia UERJ. Prof. da Disciplina de Endodontia da Universidade Federal Fluminense (UFF). Polo Universitário Nova Friburgo, Rio de Janeiro,Brasil.e-mail:cigomez@terra.com.br

***** Especialista em Endodontia (UFF).e-mail: drafernandavalle@yahoo.com.br
} 
CAMÕES IZG:

FREITAS LF

SANTIAGO CN

GOMES CC

MENEZES FV

ANÁLISE DA

CONCENTRAÇÃO

E DO PH DE

DIFERENTES

MARCAS

COMERCIAIS DE

HIPOCLORITO DE

SÓDIO

16

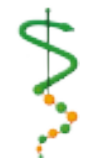

REV, ODONTOL

UNIV, C I D , S ÃO

PAULO

2012; 24(1): 15-8,

$J A N-A B R$

\section{N T R O D U ÇÃ O}

O hipoclorito de sódio, classificado como um composto halogenado, é a solução mais comumente usada na terapia endodôntica, durante o preparo químico mecânico dos canais radiculares. O simples ato mecânico de irrigação promove o controle de possível infecção superficial da polpa vital, remoção de material inorgânico, neutralização de produtos tóxicos e lubrificação dos canais radiculares (Walker ${ }^{1}, 1936$, Grossman e Meiman², 1941, Maciel ${ }^{3}, 1999$, Lopes et al. ${ }^{4}, 1999$, Cohen e Burns ${ }^{5}, 2000$, Estrela ${ }^{6}, 2004$, Imu$\mathrm{ra}^{7}, 1998$, Siqueira Júnior et al. $\left.{ }^{8}, 1999\right)$.

Segundo Maciel33, (1999), o hipoclorito a $5 \%$, por possuir uma acentuada atividade antimicrobiana e solvente de matéria orgânica, é indicado principalmente em casos de lesões periapicais. As lesões periapicais se caracterizam por um processo infeccioso de longa duração e, nessas condições, a substância irrigadora não apresenta toxicidade aos tecidos periapicais.

As soluções de hipoclorito de sódio são encontradas no comércio prontas para o uso em várias concentrações: Líquido de Dakin $0,5 \%$; Solução de Milton 1\%; Hipoclorito de sódio 2,5\%; Soda clorada 4-6\% (Cohen e Burns ${ }^{5}$, 2000, Siqueira Júnior et al. ${ }^{8}, 1999$, Milano et al. ${ }^{9}, 1991$, Leonardo $\left.^{10}, 2005\right)$.

Este trabalho visa avaliar se o hipoclorito de sódio adquirido, que permanece no consultório durante o uso, apresenta suas características quanto ao $\mathrm{pH}$ e concentração, citadas pelo fabricante, inalteradas.

Material e Método

\section{Material}

- Soda clorada lodontosul 5\% (Industrial Odontológica do Sul, Porto Alegre/RS);

- Soda clorada Asfer 2,5\% (Indústria Química Ltda, São Caetano do Sul/ $\mathrm{SP})$;

- Hipoclorito de Sódio 5,25\% (Crystal Pharm, Niterói/RJ/Brasil);

- Água Sanitária Max Globo 2,0\% à 2,5\% (W4C Indústria e Comércio Ltda, Santa Luzia - São Gonçalo/ $\mathrm{RJ})$;
- Aparelho pHagômetro pH 330i/ SET. WTW Wissenschaftlich Technische Werkstatten. Dr. Karl-Slevogt-Strabe 182362 Weilheim. Made in Germany WTW;

- Kit Hipoclorito Henrique Bassi (Easy Equipamentos Odontológicos Ltda, Belo Horizonte/MG).

- Água Destilada Asfer (Asfer Indústria Química, São Caetano do Sul/ SP).

\section{MÉ T O D O}

\section{Concentração}

Para realizar este trabalho foi utilizado o Kit Hipoclorito cujo método se baseia na redução do cloro presente na solução de hipoclorito.

Com um tubo de ensaio e um gotejador que acompanham o conjunto foram adicionadas 2 gotas à amostra a ser analisada, tomando-se o cuidado de manter o tubo na posição vertical e certificando-se de que não havia retenção da amostra nas paredes do tubo. A seguir, foram adicionadas 2 gotas da solução redutora e agitou-se o tubo, momento em que a solução adquiriu uma coloração avermeIhada. Adicionou-se, então, 2 gotas do reagente ácido e o tubo foi agitado; logo a solução adquiriu uma coloração vinho intensa. Adicionou-se 2 gotas da solução indicadora, o tubo foi agitado e a solução adquiriu a coloração escura. A seguir, efetuou-se a titulação, adicionando-se cuidadosamente o titulante gota a gota, com pequena agitação do tubo após cada adição, até a solução perder a cor escura e ficar completamente clara. O número de gotas gastas para cada teste foi anotado. A porcentagem do hipoclorito de sódio estava associada ao número de gotas utilizadas pelo titulante até a solução ficar completamente clara. Anotou-se o número de gotas gastas para cada teste e correlacionou-se com a tabela de medida da concentração fornecida pelo fabricante.

\section{$\mathrm{pH}$}

A medida dos valores do $\mathrm{pH}$ de cada solução foi realizada de acordo com o protocolo do fabricante do aparelho pH330i/SET. Media-se o pH e temperatura da solução armazenada em temperatura ambiente. O aparelho é composto por 
um sensor que quando entrava em contato com a solução dava o valor do $\mathrm{pH}$ referente. A cada troca de solução o sensor era limpo com água destilada, para, então, realizar-se uma nova medição.

\section{RESULTADOS}

Tabela 1 - Resultado da avaliação das diferentes marcas quanto à medição da concentração e $\mathrm{pH}$.

\begin{tabular}{|c|c|c|c|c|c|c|c|c|c|c|}
\hline \multirow{2}{*}{ Grupos } & \multicolumn{2}{|c|}{$\begin{array}{l}\text { Inicial } 1^{\circ} \\
\text { avaliação }\end{array}$} & \multicolumn{2}{|c|}{$\begin{array}{l}2^{\circ} \text { avaliação } \\
\left(1^{\text {a }} \text { Semana }\right)\end{array}$} & \multicolumn{2}{|c|}{$\begin{array}{l}3^{\circ} \text { avaliação } \\
\text { ( } 2^{\text {a }} \text { Semana) }\end{array}$} & \multicolumn{2}{|c|}{$\begin{array}{l}4^{\circ} \text { avaliação } \\
\text { (3 } 3^{\text {a }} \text { Semana) }\end{array}$} & \multicolumn{2}{|c|}{$\begin{array}{l}5^{\circ} \text { avaliação } \\
\text { (4 }{ }^{\text {a }} \text { Semana) }\end{array}$} \\
\hline & CONC & $\begin{array}{l}\mathrm{pH} \mathrm{a} \\
24^{\circ} \mathrm{C}\end{array}$ & CONC & $\begin{array}{l}\mathrm{pH} \mathrm{a} \\
24^{\circ} \mathrm{C}\end{array}$ & CONC & $\begin{array}{l}\mathrm{pH} \mathrm{a} \\
24^{\circ} \mathrm{C}\end{array}$ & CONC & $\begin{array}{l}\mathrm{pH} \mathrm{a} \\
24^{\circ} \mathrm{C}\end{array}$ & CONC & $\begin{array}{l}\mathrm{pH} \mathrm{a} \\
24^{\circ} \mathrm{C}\end{array}$ \\
\hline $\begin{array}{c}\text { Gl } \\
(5,25 \%)\end{array}$ & $4 \%$ & 11,8 & - & 11,9 & $3,50 \%$ & 11,7 & - & 11,7 & $3,25 \%$ & 11,7 \\
\hline G II (5\%) & $3 \%$ & 11,7 & - & 11,7 & $3 \%$ & 11,8 & - & 11,8 & $3 \%$ & 11,8 \\
\hline $\begin{array}{c}\text { G III } \\
(2,5 \%)\end{array}$ & $2,25 \%$ & 11,7 & - & 11,7 & $2,25 \%$ & 11,5 & - & 11,8 & $2,25 \%$ & 11,5 \\
\hline $\begin{array}{c}\text { G IV } \\
(2-2,5 \%)\end{array}$ & $2,25 \%$ & 11,5 & - & 11,5 & $2,25 \%$ & 11,5 & - & 11,5 & $2 \%$ & 11,5 \\
\hline
\end{tabular}

GI - Hipoclorito de Sódio 5,25\% Crystal Pharm

Gll- Soda Clorada lodontosul 5\%

GIII- Soda Clorada Asfer 2,25\%

GIV- Água Sanitária Max Globo 2-2,5\%

(-) Não coletadas na semana.

\section{I SCUSSÃO}

As melhorias constantes na Endodontia, não apenas por meios e técnicas de instrumentação, como também, no uso de substâncias químicas auxiliares que promovam de forma efetiva o saneamento e a descontaminação do complexo sistema de canais radiculares, justificam o propósito de uma pesquisa que busque a análise da concentração e do pH das substâncias químicas utilizadas na irrigação durante a terapia endodôntica (Lopes et al. ${ }^{4}, 1999$, Ingle ${ }^{11}, 1989$, Jeansonne e White ${ }^{12}, 1994$, Marchesan et al. ${ }^{13}$, 1998).

É sabido que as soluções de hipoclorito de sódio são eficazes como substâncias antimicrobianas. Porém sua concentração apresenta instabilidade. A importância do uso do hipoclorito de sódio como substância química irrigadora, durante o tratamento endodôntico, fez com que estudos das diferentes concentrações fossem realizados para avaliar sua eficácia e instabilidade (Gomes ${ }^{14}, 2007$, Bairan e Caldera ${ }^{15}$, 2000, Hulsmann e Hahn ${ }^{16}$, 2000, Soares e Goldberg ${ }^{17}$, 2001, Noites et al. ${ }^{18}$, 2009, Lopes ${ }^{19}$, 2010). hipoclorito de sódio utilizado em procearmazanamento e embalagem da solição podem reduzir o teor de cloro ativo e, consequentemente, seus efeitos esperados.

No presente trabalho, pôde-se observar que, das substâncias irrigantes utilizadas na pesquisa, apenas a marca Max Globo se manteve fiel quanto à concentração descrita pelo fabricante.

Outro fator a ser considerado diz respeito à substância manipulada, muitas vezes é a opção de escolha para irrigação dos canais radiculares. Porém, de acordo com a presente pesquisa, essa substância não se manteve fiel quanto à concentração descrita.

\section{CONCLUSÃO}

A interpretação dos resultados aponta para o fato de que, em todas as substâncias químicas avaliadas, o $\mathrm{pH}$ se manteve constante durante a pesquisa. Em contrapartida, somente a concentração da Água Sanitária Max Globo se manteve fiel, de acordo com a concentração estabelecida pelo fabricante. dimentos endodônticos. As condições de
A escolha das substâncias testadas foi de acordo com as marcas comerciais mais usadas por Cirugiões-Dentistas em geral.

Resultados semelhantes ao do presente trabalho foram encontrados por Gomes ${ }^{14}$ (2007), que analisou a concentração do

CAMÕES IZG

FREITAS LF

SANTIAGO CN

GOMES CC

MENEZES FV

ANÁLISE DA

CONCENTRAÇÃO

E DO PH DE

DIFERENTES

MARCAS

COMERCIAIS DE

HIPOCLORITO DE

SÓDIO
REV, ODONTOL. UNIV, CID, SÃO PAULO

2012; $24(1): 15-8$, JAN - ABR 
CAMÕES IZG

FREITAS LF

SANTIAGO CN

GOMES CC

MENEZES FV

ANÁLISE DA

CONCENTRAÇÃO

E DO PH DE

DIFERENTES

MARCAS

COMERCIAIS DE

HIPOCLORITO DE

SÓDIO

18

REV, ODONTOL.

UNIV, CID, SÃO PAULO

2012; 24(1): 15-8,

$J A N-A B R$

\section{REFERÊNCIAS}

1. Walker AA. Definite and dependable therapy for pulpless teeth. J Am Dent Assoc 1936 Aug;23(2):1418-24.

2. Grossman LI, Meiman BW. Solution of pulp tissue by chemical agents. J Am Dent Assoc 1941 Feb.;28(1):223-5.

3. Maciel AAC. Manual de endodontia. Rio de Janeiro: Guanabara Koogan; 1999.

4. Lopes HP, Martins CMMS, Carvalho RdM, Siqueira Júnior JF, Cabreira MS. Análise de diversas marcas de água sanitária: confiabilidade e segurança para uso como solução química auxiliar em endodontia Rev bras odontol 1999 nov.-dez;56(6):319-22.

5. Cohen S, Burns R. Caminhos da polpa. 7. ed. Rio de Janeiro: Guanabara Koogan; 2000.

6. Estrela C. Ciência endodôntica. São Paulo: Artes Médicas; 2004.

7. Imura N. Endodontia para o clínico geral. São Paulo: Artes Médicas; 1998.

8. Siqueira Júnior JF, Moraes SR, Lopes HP. Atividade antimicrobiana de águas sanitárias disponíveis no mercado nacional. Rev bras odontol 1999 mar.-abr;56(2):57-60.

9. Milano NF, Girardi V, Bergold AM, Chiapini LG. Alguns aspectos do uso do hipoclorito de sódio em Endodontia Rev Fac Odontol Porto Alegre 1991 jul.;32(1):7-10.

10. Leonardo MR. Endodontia: tratamento de canais radiculares: princípios técnicos e biológicos. São Paulo Artes Médicas; 2005.

11. Ingle JI. Endodontia. 3. ed. Rio de Janeiro: Interamericana; 1989.

12. Jeansonne MJ, White RR. A comparison of $2.0 \%$ chlorhexidine gluconate and 5.25\% sodium hypochlorite as antimicrobial endodontic irrigants. J Endod 1994 Jun;20(6):276-8.

13. Marchesan MA, Souza RA, Guerisoli DMZ, Silva RS, Pécora JD. Análise de algumas propriedades físico-químicas das águas sanitárias encontradas no mercado brasileiro Rev bras odontol 1998 set.-out.;55(5):301-3.

14. Gomes MCP. Análise da real concentração de cloro ativo em soluções de hipoclorito de sódio [Trabalho de Conclusão de Curso de Odontologia]. São Paulo: Universidade Cruzeiro do Sul; 2007. [Acesso em] Disponível em: http://www.endonline. com.br/artigos/tcc/mcarmo/mcarmo.htm.

15. Bairan E, Caldera M. Uma visão atualizada do uso do hipoclorito de sódio na endodontia. 2000 [Acesso em 17/01/09]; Disponível em: http://www.carlosboveda. com.br.

16. Hulsmann M, Hahn W. Complications during root canal irrigation--literature review and case reports. Int Endod J 2000 May;33(3):186-93.

17. Soares IJ, Goldberg F. Endodontia: técnicas e fundamentos. Porto Alegre: Artmed; 2001.

18. Noites R, Carvalho MF, Vaz IP. Complicações que podem surgir durante o uso do hipoclorito de sódio no tratamento endodôntico. Rev Port Estomatol Cir Maxilofac 2009 50(1):53-6.

19. Lopes HP. Endodontia: biologia e técnica. 3. ed. Rio de Janeiro: Guanabara Koogan; 2010.

Recebido em: 01/08/2011

Aceito em: 15/09/2011 